\title{
Gesellschaft und Politik
}

\section{Zur Einführung}

Die jüngere Vergangenheit Brasiliens hatte Anfang der 80er Jahre recht verheißungsvoll begonnen. Die Militärs waren nach rund zwanzig Jahren schließlich an ihrer eigenen Inkompetenz und an dem Widerstand der Gesellschaft gescheitert. Neuartige gesellschaftliche Organisationsformen hatten sich herausgebildet oder ältere hatten einen neuen Sinn bekommen: Arbeiter hatten sich mit gemäßigt sozialistischen Intellektuellen in der ersten Programm-Partei Brasiliens zusammengetan, neue kirchliche Vereinigungen in Gestalt der Basisgemeinden und der Landarbeiter-Seelsorge waren entstanden und hatten eine theologische Rechtfertigung in der Befreiungstheologie gefunden, soziale Bewegungen von Stadtteilbewohnern und Hausfrauen, gelegentlich durch Universitätsstudenten und -dozenten, darunter erstmals auch Feministinnen, unterstützt, hatten ihre Anliegen durchsetzen können. Auch traditionellere, eher berufsständische Verbände wie der Anwaltsverein, die Gesellschaft für den Fortschritt der Wissenschaft, Journalisten, Unternehmer und viele andere mehr hatten sich wirkungsvoll manifestiert.

In dem Bemühen, das Neue auf einen Begriff zu bringen und die Richtung anzudeuten, die nun einzuschlagen war, um die Repression endgültig zu überwinden und die Partizipation aller Brasilianer an politischen Entscheidungen in einer Nova República sicherzustellen, nannten Sozialwissenschaftler den Bereich der neuartigen Opposition die zivile Gesellschaft im Unterschied zu dem militärisch-technokratischen Komplex, den es in die Schranken zu weisen galt, und zur politischen Gesellschaft, die als belastet und korrumpiert angesehen wurde.

Der Begriff von der zivilen Gesellschaft machte Karriere, aber nicht mit einer der Bedeutungen, die sich bei Platon, Hegel, Locke, Rousseau und anderen Klassikern finden, sondern auf der Grundlage der Definition des italienischen Kommunisten Antonio Gramsci. Vermutlich auch deswegen, weil sie in einer romanischen Gesellschaft entstanden war und weil sie als Teil der zivilen Gesellschaft die Figur des organischen Intellektuellen herausstellte, einer mit den Arbeitern sozusagen organisch verbundenen, aber von der Handarbeit befreiten Person, die als Sprecher der Volks- 
massen legitimiert ist und mit der sich viele brasilianische Intellektuelle wohl identifizieren wollten. Der als Wissenschaftler und Politiker gleichermaßen bedeutende Soziologe Fernando Henrique Cardoso hat die brasilianische Rezeption von Gramsci später als fehlerhaft kritisiert: »It was discovered, without anyone having called it scandalous, that what was happening here was the reverse of what Gramsci described with relation to Italy. «1

Die zivile Gesellschaft wurde zum Kampfbegriff ${ }^{2}$, verlor damit analytische Potenz, wie überhaupt das begriffliche Triptychon als Abbild der Gesellschaft viel zu simpel war; und das hatte für die weitere Entwicklung böse Folgen.

Im Zuge der Entmilitarisierung des brasilianischen Staatsapparats mußten in kurzer Zeit viele Machtpositionen neu besetzt werden. Dafür standen aus der politischen Gesellschaft nur einige Randständige bereit, so zum Beispiel ein junger Lokalpolitiker aus einem der kleinen nordöstlichen Bundesstaaten, Fernando Collor de Mello, der später zum Präsidenten der Republik gewählt wurde, oder der sehr alte Ulysses Guimarães, dem es gelungen war, auch in der artifiziellen Opposition der Militärregierungen eine gewisse Autonomie zu bewahren. Die meisten Positionen nahm jedoch eine Generation von Menschen ein, die sich in den vergangenen zwanzig Jahren, vielfach und zwar unfreiwillig im Ausland ausgebildet hatte, ohne sich in friedlichen politischen Prozessen erproben zu können. Sie zählten sich zur zivilen Gesellschaft oder ließen sich dazu zählen und meinten, gegen die Anfechtungen der politischen Gesellschaft gefeit zu sein und die traditionellen Formen des Gewinnens, Bewahrens und Verlierens politischer Macht ignorieren oder naiv gebrauchen zu können.

Zwar hatten die Militärs in der Endphase ihrer Herrschaft Wirtschaft und Staatsapparat ruiniert und ihren zivilen Nachfolgern dadurch eine riesige Erblast hinterlassen, auch verschärfte sich die Belastung durch die Auslandsverschuldung und den aggressiven internationalen Wirtschaftswettbewerb, aber die neue Generation ziviler Politiker und Beamten zeichnete sich eben auch durch Inkompetenz ${ }^{3}$ und durch Anfälligkeit für Nepotismus, Klientelismus und Korruption aus. Ein Paradebeispiel für ihr Versagen war die Wirtschaftsministerin, Zélia Cardoso de Mello, vormals Professorin der angesehenen Universität von São Paulo, die mit politischer Naivität einen massiven Eingriff in die Wirtschaft durchsetzte, sich dabei persönlich bereicherte und bei einer Affäre mit dem Justizminister sowohl die Auswirkungen auf die Gruppendynamik des Ministerkabinetts als auch die Agressivität einer selbstbewußt gewordenen Presse unterschätzte.

Ungenaues Denken der Intellektuellen und unverantwortliches Handeln der neuen Generation von Politikern und Administratoren dürfen freilich nicht darüber hinwegtäuschen, daß die Gesellschaft sich unter der Militärherrschaft geändert hatte wie nie

1 Cardoso 1989: $318 f$.

2 Man kann, wie Bernardo Sorj (1990: 111f.), auch Sociology of Desire sagen.

3 Singer 1988. 
zuvor in zwei Jahrzehnten. ${ }^{4}$ Das kurze brasilianische Wirtschaftswunder und einige wenige, kaum noch umkehrbare Rationalisierungsprozesse in Wirtschaft und öffentlicher Verwaltung, hatten dazu ebenso beigetragen, wie der schon erwähnte Widerstand gegen die Diktatur. Ein Indiz für diesen Wandel kann man schon in der Tatsache erkennen, daß die Gesellschaft fähiger geworden ist, auf Krisen elastisch zu reagieren; wieviel weniger dramatisch war die Situation, die 1964 ausreichte, um eine Militärdiktatur zu errichten, als manche bittere Not, die seither ausgebrochen ist. Aus den Wahlen des Jahres 1994 kann eine politische Erneuerung hervorgehen; die hohen Erwartungen an die Funktionsfähigkeit eines demokratischen Prozesses unter einer 1988 mit beispielhafter Partizipation erarbeiteten neuen Verfassung bestimmten 1993 den politischen Diskurs allerdings so sehr, daß man leider auch damit rechnen muß, sie könnten aufs Neue enttäuscht werden.

Alle Beiträge in diesem Kapitel beschäftigen sich mehr oder weniger intensiv mit dem Wandel, in dem sich Brasiliens Gesellschaft und Politik befindet. Zunächst stellt Renate Rott die veränderten und sich noch wandelnden Familienformen und $\mathrm{Ge}$ schlechterbeziehungen dar. Ihr folgt Detlev Schelsky mit einer Darstellung der ebenfalls gewandelten Situation der ethnischen Minderheiten. Nachdem Volker Lühr die soziale Ungleichheit im veränderten Selbstbild der brasilianischen Gesellschaft dargestellt hat, folgt meine Einschätzung der Änderungen in der Sozialstruktur Brasiliens und derjenigen in der politischen Kultur durch Gilberto Calcagnotto. Das herausragende Ereignis der Redemokratisierung war die Verfassung von 1988, die Wolf Paul vorstellt; wie sehr freilich Rechtspflege und öffentliche Verwaltung noch reformiert werden müssen, geht aus den Beiträgen von Hans-Joachim Henckel und Ingo Schultz hervor. Die Folge von Beiträgen schließt mit einer Einschätzung der neuen Rolle des Militärs durch Bernhard Moltmann und der brasilianischen Außenpolitik durch Stefan A. Schirm.

Weil nicht wenige Quellen von mehreren Autoren benutzt wurden, ist für diesen Teil des Buches eine gemeinsame Bibliographie erstellt worden; sie enthält auch Hinweise auf weiterführende Literatur. - Die Manuskripte wurden im Herbst 1993 abgeschlossen. Dieser Hinweis ist unentbehrlich, wenn Wandel der gemeinsame Nenner aller Beiträge ist.

4 Almeyda/Sorj 1983. 